\title{
O que é um Estudo de Caso e quais as suas potencialidades?
}

\section{What is a Case Study and what are its potentialities?}

\section{¿Qué es un estudio de caso y cuáles son sus potencialidades?}

\author{
iD Natália Guimarães Duarte Sátyro \\ Universidade Federal de Minas Gerais, Belo Horizonte, Minas Gerais, Brasil \\ nsatyro@gmail.com \\ (iD) Raquel Wanderley D'Albuquerque \\ Universidade Federal de Minas Gerais, Belo Horizonte, Minas Gerais, Brasil \\ raquel.dalbuquerque@gmail.com
}

Resumo: Este trabalho tem como objetivo central fazer uma discussão acerca da compreensão dos desenhos de estudos de caso, a fim de destacar suas vantagens e desafios de aplicação na Ciência Política. Parte-se de uma discussão conceitual sobre o que são estudos de caso em suas diversas definições e concepções na literatura sobre metodologia em Ciências Sociais, a fim de propor uma distinção necessária entre a estratégia de pesquisa, o número de casos e o número de observações. Aborda-se o papel dos estudos de caso no tratamento da multicausalidade e da complexidade causal dos fenômenos sociais, discutindo em termos de validade interna e externa e de estratégias de análise causal. Nesse sentido, argumenta-se a favor de uma diferente perspectiva ao entendimento mais comum dos estudos de caso: em vez de se restringir aos desenhos que se estruturam a partir de uma ou poucas observações pela via qualitativa, também é possível compreendê-los como estratégia de pesquisa que adota modelos de análise em- 
pírica utilizando técnicas quantitativas a partir do estabelecimento de variações internas aos casos. Por fim, argumenta-se que as diferentes lógicas de causação e a característica densa dos estudos de caso atribuem rigor metodológico e um relevante papel na construção e no teste de teorias na Ciência Política.

Palavras-chave: Estudo de Caso. Multicausalidade. Validade. Ciência Política.

Abstract: The aim of this paper is to discuss the understanding of case study research design, in order to evidence its advantages and application challenges in Political Science. It starts with a conceptual discussion about the different definitions and conceptions about case studies found in the literature and then distinguish research strategies from number of cases and number of observations. We approach the role of case studies in treating multi-causality and the causal complexity of social phenomena, discussing in terms of internal and external validity and causal analysis strategies. In this sense, argumentation in favor of a different perspective is more common in case studies: instead of restricting designs that are structured in one or few observations treated in a qualitative approach, it is also possible to understand it as research strategies that ad opt models of empirical analysis using quantitative techniques from the variation within the case. Finally, it is argued that different logics of causation and a dense characteristic of case studies give the methodological rigor and a relevant place in creating and testing theories in Political Science.

Keywords: Case Study. Multi-causality. Validity. Political science.

Resumen: Este trabajo tiene como objetivo central hacer una discusión sobre la comprensión de los diseños de estudios de caso, a fin de destacar sus ventajas y desafíos de aplicación en la Ciencia Política. Se parte de una discusión conceptual sobre lo que son estudios de caso en sus diversas definiciones y concepciones en la literatura sobre metodología en Ciencias Sociales, a fin de proponer una distinción necesaria entre la estrategia de investigación, el número de casos y el número de observaciones. Se discute el pa- 
pel de los estudios de caso en el tratamiento de la multicausalidad y de la complejidad causal de los fenómenos sociales, discutiendo en términos de validez interna y externa y de estrategias de análisis causal. En este sentido, se argumenta a favor de una diferente perspectiva al entendimiento más común de los estudios de caso: en vez de restringirlos a los diseños que se estructuran a partir de una o pocas observaciones por la vía cualitativa, también es posible comprenderlos como estrategia de investigación que adopta modelos de análisis empíricos utilizando técnicas cuantitativas a partir del establecimiento de variaciones internas a los casos. Por último, se argumenta que las diferentes lógicas de causación y la característica densa de los estudios de caso los atribuyen rigor metodológico y un relevante papel en la construcción y en la prueba de teorías en la Ciencia Política.

Palabras clave: Estudio de caso. Multicausalidad. Validez. La ciencia política.

Data de recebimento do artigo: 30/10/2018

Data de aprovação do artigo: 05/06/2019 


\section{Introdução}

Dentre as diversas formas de combinar pergunta, base teórica, dados e métodos - os elementos principais do desenho de pesquisa - têm-se em mãos várias possibilidades de estratégias de investigação que podem ser utilizadas, a depender, primeiramente, da pergunta que está sendo proposta e de vários fatores que dependerão dela e do que é necessário para investigá-la. Alguns fenômenos apenas serão analisados corretamente de maneira compreensiva, intensiva e densa: são aqueles que demandam um estudo de caso.

Os estudos de caso constituem uma dentre as várias estratégias de pesquisa existentes e, como tal, apresentam características, vantagens e limitações próprias, sendo mais apropriados em certas condições. O objeto deste trabalho é uma compreensão das características, possibilidades e desafios do desenho de estudo de caso, sem pretender a exaustão. Aqui não se pretende trazer uma visão inovadora ou mesmo uma contribuição inédita ao debate como a que foi feita por trabalhos recentes, como o de Rezende (2017). Ao contrário, o objetivo, mais modesto, é de preencher a lacuna de textos em português que tragam o debate recente e, portanto, possam contribuir para a entrada neste debate do leitor iniciante: seja aquele com uma formação mais quantitativa que carrega uma série de preconceitos com relação às abordagens qualitativas, seja aquele que se identifica mais com essa abordagem, mas ainda está em seus primeiros passos.

O trabalho se estrutura em quatro seções, além desta introdução: primeiramente, faz-se uma discussão das visões de diversos autores sobre a definição de estudos de caso e sua caracterização; em seguida, discutem-se os desafios e possibilidades desse tipo de desenho, com destaque para a questão da multicausalidade e da validade. Daí parte-se para uma discussão de exemplos clássicos e contemporâneos do uso do desenho na Ciência Política e, por fim, apresentam-se as considerações finais em uma perspectiva que vai à defesa dos estudos de caso como desenhos que permitem 
O que é um Estudo de Caso e quais as suas potencialidades?

Natália Guimarães Duarte Sátyro•Raquel Wanderley D’Albuquerque

tanto gerar como testar teorias, desde que sejam apreendidos em sua completude.

\section{Definindo Estudo de Caso}

Um estudo de caso é de grande utilidade para compreender fenômenos sociais complexos que demandem uma investigação que preserve suas características holísticas e desvende os processos e mecanismos significativos, separando-os de um grande conjunto de fatores e processos secundários ao processo central da análise. Entretanto, quase sempre é necessária uma abordagem pluralística de diferentes estratégias de pesquisa, de modo a entender os propósitos exploratórios, descritivos ou explicativos de forma sobreposta e complementar e não hierárquica (YIN, 2001). Dessa forma, defendemos que a diferenciação das estratégias de pesquisa se dá (i) pela natureza da pergunta de pesquisa, aqui diretamente ligada à natureza do seu objeto de pesquisa (YIN, 2001), (ii) pelo grau de controle que se tem sobre os eventos, processos, mecanismos ou fatores relevantes e (iii) pelo foco nas causas ou nos efeitos do fenômeno central de interesse - esses dois últimos defendidos por Rezende (2017) ${ }^{1}$.

Primeiramente, é necessário esclarecer algumas diferenças fundamentais entre os estudos de casos e os métodos comparativos e qualitativos de forma geral, que são comumente submetidos a uma confusão de interpretações. O método comparativo diz respeito ao uso de comparações entre certo número de casos a fim de inferir sobre algum fator comum a eles, enquanto o método qualitativo consiste em uma estratégia de coleta e análise de dados que pode ser empreendida em diferentes desenhos de pesquisa,

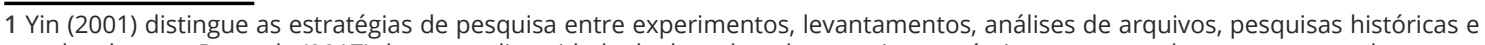
estudos de caso. Rezende (2017) destaca a diversidade de desenhos de pesquisa possíveis que se complementam e que devem ser adequados e calibrados para produzir inferências válidas. A tipologia do pluralismo inferencial proposta pelo autor sugere que os desenhos de pesquisa variam em termos de sua lógica básica (com foco na causa dos efeitos ou no efeito das causas) e conforme maior ou menor grau de controle. Com isso, podemos classificar os desenhos como análises descritivas (baixo controle e foco nas causas dos efeitos), explicação causal (baixo controle e foco nos efeitos das causas), interpretação causal (alto controle e foco nas causas dos efeitos) e estimação causal (alto controle e foco nos efeitos das causas). 
que serão exemplificados no decorrer do texto. Já os estudos de caso envolvem a ideia de examinar um único caso internamente. Entretanto, pode-se defini-los com base em análises internas de um único caso ou comparações de um pequeno número de casos, como esclareceremos mais adiante. Essa combinação consiste na forma mais forte de gerar inferências a partir de estudos de caso, inclusive com a possibilidade de gerar teorias (GEORGE e BENNETT, 2004). Além disso, apesar de ser normalmente classificado como uma abordagem qualitativa, ele não se restringe a ela, podendo ser quantitativo. Nesse caso, o que o distingue é a intensidade de estudo de um fenômeno, não a natureza da abordagem utilizada.

A estratégia metodológica do estudo de caso tem como base questões de pesquisa tanto do tipo "qual" ou "como", que podem gerar análises descritivas inferenciais, quanto do tipo "por que", de natureza explicativa. De acordo com Yin (2001), pode-se ter foco tanto em eventos comportamentais que não terão grandes exigências de controle, mas também sobre os processos de distintas naturezas (sociais, políticos etc.) ou conjunções de processos - fenômenos nos quais diversos mecanismos causais de diferentes áreas e dimensões produzem determinados resultados. Naturalmente, eventos dessa natureza necessitam da análise de uma ampla variedade de evidências - documentos, artefatos, entrevistas e observações (YIN, 2001). Yin (2001, p. 32 e 33) defende que um estudo de caso

investiga um fenômeno contemporâneo dentro de seu contexto da vida real, especialmente quando os limites entre o fenômeno e o contexto não estão claramente definidos. (...) [Este] enfrenta uma situação tecnicamente única em que haverá muito mais variáveis de interesse do que pontos de dados, e, como resultado, baseia-se em várias fontes de evidências, com os dados precisando convergir em um formato triângulo, e, como outro resultado, beneficia-se do desenvolvimento prévio de proposições teóricas para conduzir a coleta e a análise de dados. 
O desenho de "estudo de caso" deveria ser entendido como "estudo de um fenômeno" para minimizar a confusão. Concordamos em afirmar que se trata de um $n=1$ se, e somente se, o $n$ se referir a um fenômeno, não a um caso, pois o "estudo de caso" (que representa um fenômeno) pode, de fato, se referir somente a um caso (como unidade de análise), mas poderá envolver mais de um caso (sempre poucos). No entanto, qualquer que seja a unidade de análise, o estudo sempre será baseado em muitas observações e evidências (que garantirão a variação mínima necessária ao estudo de qualquer fenômeno). Assim, para nós, só é possível tratar de estudo de caso como $n=1$ quando o $n$ se refere ao fenômeno estudado e não ao caso estudado ou ao número de observações envolvidas.

Defendemos a possibilidade de classificação de estudos de caso segundo, pelo menos, duas perspectivas: (i) segundo a intensidade da análise ou (ii) segundo o número de casos. Pela primeira vertente, um estudo de caso se configura pela análise densa, holística e com foco nos processos ou conjunções de processos internos ao caso, com ganhos em intensidade e conhecimento dos mecanismos de funcionamento do caso, quase sempre realizado de forma aprofundada², compreensiva e densa.

Já de acordo com a segunda perspectiva, um estudo de caso pode ocorrer quando o fenômeno estudado se refere a apenas um caso (um país, por exemplo) e, dentro dele, analisam-se as variações, podendo ser menos denso, buscando entender tendências gerais e efeitos do funcionamento do caso, geralmente pela via quantitativa - essa perspectiva define estudo de caso em oposição ao estudo cross-case em que se utiliza mais de um caso para fazer comparações entre sistemas, por exemplo. Cabe ainda destacar a existência de um trade-off entre o maior número de casos (ou variações internas ao caso) e a profundidade e densidade de tratamento do fenômeno estudado, sendo que quando se opta por uma dimensão, perde-se na outra. Ou seja, aqui se trata do que Sartori (1970) chama de graus distintos de abstração.

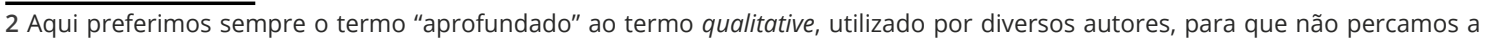
ideia de que é possível realizar estudos de caso baseados em dados quantitativos, exemplo, Sátyro et al (2016). 
Estudo de caso é facilmente confundido com estudo cross-case. Um estudo de caso só pode conter vários casos quando o fenômeno em foco engloba/atravessa vários casos, ou seja, os casos são parte dele. Por exemplo: uma pesquisadora pode querer estudar uma guerra ou um conflito regional que envolveu vários países, isto é um estudo de caso. Outra pesquisadora que quer comparar os diversos conflitos de natureza similar que ocorrem em vários países está fazendo um estudo cross-case, não um estudo de caso.

Gerring (2007) mostra uma gama de definições e características encontradas na literatura para definir um estudo de caso. No entanto, o autor opta pelo que chama de uma definição mínima. Para ele, uma estratégia de pesquisa do tipo estudo de caso corresponde ao "estudo intensivo de um único caso com o propósito de compreender a classe maior de casos (a população)" (GERRING, 2007 p. 95. Tradução livre). Além disso, destaca que é possível que um estudo de caso incorpore mais de um caso, entretanto, como já mencionado, isso implica um trade-off, uma vez que, em certo ponto, não é mais possível manter a intensidade de análise dos casos. Sendo assim, quando a ênfase do estudo passa de um caso individual para uma amostra de casos, temos um estudo do tipo cross-case.

Aqui, reafirmamos nossa postura que diferencia cross-case de estudo de caso de algum fenômeno ou evento que engloba vários casos. Isso não nos isenta da importância de mostrar o quanto é relevante analisar as afinidades entre os dois métodos, inclusive para diferenciá-los. É ilustrativo o exercício feito por Gerring (2007) sobre isso. Com base em sua definição, o autor elenca oito possíveis afinidades metodológicas dos estudos de caso e dos estudos cross-case, sob as dimensões dos objetivos da pesquisa e formato do universo empírico, como presentes no quadro 1. Essas afinidades são entendidas como atributos do estudo de caso que, frequentemente, mas não sempre, são associados com os estudos de caso, mas não são definidoras dele - visto que o autor já adotou uma definição mínima anteriormente. 
Quadro 1 - Afinidades Metodológicas: Estudo de Caso x Estudo Cross-Case

\begin{tabular}{|c|c|c|c|}
\hline & & \multicolumn{2}{|c|}{ Afinidades Metodológicas } \\
\hline & & Estudo de Caso & Estudo Cross-Case \\
\hline \multirow{4}{*}{$\begin{array}{c}\text { Objetivos } \\
\text { da } \\
\text { Pesquisa }\end{array}$} & Hipótese & Gerar hipóteses & Testar hipóteses \\
\hline & Validade & Prioriza validade interna & Prioriza validade externa \\
\hline & Insight Causal & Foca nos mecanismos causais & Foca nos efeitos causais \\
\hline & Abrangência & $\begin{array}{l}\text { Inferências profundas e } \\
\text { intensas }\end{array}$ & Inferências amplas \\
\hline \multirow{4}{*}{$\begin{array}{l}\text { Fatores } \\
\text { Empíricos }\end{array}$} & $\begin{array}{l}\text { População de } \\
\text { Casos }\end{array}$ & $\begin{array}{l}\text { Maior grau de heterogenei- } \\
\text { dade }\end{array}$ & $\begin{array}{l}\text { Maior grau de homogenei- } \\
\text { dade }\end{array}$ \\
\hline & Força Causal & Causalidade mais forte & Causalidade mais fraca \\
\hline & Variação Útil & Raramente varia (small-n) & Comumente varia (large- $n$ ) \\
\hline & Dados Disponíveis & Concentração dos dados & Dispersão dos dados \\
\hline
\end{tabular}

Fonte: Adaptado de Gerring (2007, p. 346).

Ao enfrentar os trade-offs de posicionamento das afinidades para cada uma das dimensões, um estudo de caso se depara com possibilidades e limitações. Como é mais comum o posicionamento desse tipo de desenho com a primeira coluna de afinidades, um estudo de caso é aquele que gera hipóteses, prioriza validade interna, tem foco principal na identificação de processos e mecanismos que expressam fortes relações de causalidade e aparecem via inferências profundas e intensas a partir da análise de dados concentrados. Entretanto, conta com uma baixa variação útil, devido ao pequeno número de casos, o que leva à menor força da validade externa e dificulta o teste de hipóteses ou identificação de efeitos causais para realizar inferências amplas (GERRING, 2007). Nessa perspectiva, os estudos de casos são melhor aproveitados quando a estratégia de pesquisa adotada é exploratória (e aqui discordamos frontalmente desta abordagem). 
Dois aspectos devem ser ressaltados para essa construção conceitual. Primeiro, consideramos a comparação entre casos como secundária, pois defendemos que quando a comparação entre casos for o foco temos um estudo cross-case, não um estudo de caso, pois se trata de mais de um fenômeno em pauta. Outro ponto é a diferença entre análise intensiva e exaustiva. Em geral, quando tratamos de análises exaustivas nos referimos àquelas em que a pesquisadora tenta nos dizer, ou analisar, absolutamente todos os fatores ou processos dentro de um fenômeno. Isso não é um procedimento correto, pois se uma pesquisadora diz que tudo é importante de se analisar, significa que não há um guia teórico-analítico para informá-la do que é relevante ou não dentro daquele processo. Ou seja, quando tudo é importante, os processos, as evidências realmente importantes perdem centralidade na análise e isso não é o desejável.

Informações contextuais são de extrema importância, uma vez que necessitamos de um contexto específico no qual possamos compreender o processo causal analisado. Mas é necessário que a pesquisadora justifique teórica ou empiricamente porque aquele contexto é importante e outros contextos não o são. Isso porque, afinal, aqui defendemos também pesquisas orientadas por teoria e se o contexto é o centro (e não os fatores guiados teoricamente), temos ou um problema teórico ou um problema de desenho inadequado.

Outro aspecto importante para definir esse tipo de desenho é o julgamento precipitado que alguns pesquisadores proferem ao dizer que não se pode testar ou gerar teorias a partir de estudos de caso pelo simples fato de serem baseados em apenas um caso. Aqui defendemos que essas são proclamações precipitadas, pois tratamos de um caso, não de uma observação. Caso esse inclusive que, como afirma De Vaus (2001), com a replicação em condições distintas que apresentem resultados consistentes, torna-se possível atribuir maior confiança aos resultados encontrados. $\mathrm{Na}$ Ciência Política temos exemplos clássicos dessa possibilidade, a começar pelo estudo de New Haven por Robert Dahl, que gera sub- 
sídios para a formulação de uma teoria geral sobre democracia. Repetindo, isso nos ensina que estudo de caso não é estudo de uma observação ou de uma evidência, mas da multiplicidade de implicações observáveis dentro de um caso. Evidências essas que, se bem costuradas, podem gerar ou testar teorias.

De Vaus (2001) propõe uma forma de pensarmos os diferentes tipos de estudos de caso a partir da combinação entre diferentes elementos que compõem sua natureza: temporalidade, ordenamento dos casos, tratamento da teoria, relação das unidades de análise e número de casos. Segundo o autor, combinações entre esses fatores resultam em 64 possibilidades lógicas diferentes para compor um desenho de estudo de caso, cada uma com formas distintas de condução, análise e coleta de dados. No quadro 2 é possível encontrar as variações possíveis dos desenhos de estudo de caso, segundo o autor.

\section{Quadro 2 - Variações dos Desenhos de Estudo de Caso}

\begin{tabular}{|c|c|c|c|c|c|c|c|c|c|c|}
\hline \multirow{4}{*}{\multicolumn{3}{|c|}{$\begin{array}{r}\text { NATUREZA } \\
\text { TEMPORALIDADE } \\
\text { ORDEM DOS CASOS }\end{array}$}} & & & & & & & & \\
\hline & & & \multicolumn{4}{|c|}{ DESCRITIVA } & \multicolumn{4}{|c|}{ EXPLICATIVA } \\
\hline & & & \multicolumn{2}{|c|}{ RETROSPECTIVA } & \multicolumn{2}{|c|}{ PROSPECTIVA } & \multicolumn{2}{|c|}{ RETROSPECTIVA } & \multicolumn{2}{|c|}{ PROSPECTIVA } \\
\hline & & & Paral. & Sequenc. & Paral. & Sequenc. & Paral. & Sequenc. & Paral. & Sequenc. \\
\hline \multirow{4}{*}{$\begin{array}{l}\text { Único } \\
\text { Caso }\end{array}$} & \multirow{2}{*}{$\begin{array}{c}\text { Unidades } \\
\text { Incorporadas }\end{array}$} & Gerar & & & & & & & & \\
\hline & & Testar & & & & & & & & \\
\hline & \multirow{2}{*}{$\begin{array}{l}\text { Unidades } \\
\text { Holísticas }\end{array}$} & Gerar & & & & & & & & \\
\hline & & Testar & & & & & & & & \\
\hline \multirow{4}{*}{$\begin{array}{l}\text { Múl- } \\
\text { tiplos } \\
\text { Casos }\end{array}$} & \multirow{2}{*}{$\begin{array}{c}\text { Unidades } \\
\text { Incorporadas }\end{array}$} & Gerar & & & & & & & & \\
\hline & & Testar & & & & & & & & \\
\hline & \multirow{2}{*}{$\begin{array}{l}\text { Unidades } \\
\text { Holísticas }\end{array}$} & Gerar & & & & & & & & \\
\hline & & Testar & & & & & & & & \\
\hline
\end{tabular}

Fonte: Adaptado de De Vaus (2001, p. 229). 
Assim, um estudo de caso pode assumir uma postura (i) descritiva ou explicativa, (ii) de construção ou de teste de teorias, (iii) com um ou múltiplos casos, (iv) com unidade de análise tratada incorporada ou holisticamente, (v) de modo sequencial ou paralelo, (vi) retrospectivo ou prospectivo. Portanto, segundo essa perspectiva, um desenho que contemple um único caso, cujas unidades de observação são analisadas de forma holística com o objetivo descritivo e de construção de teorias, é considerado um estudo de caso. De forma semelhante, um desenho que tenha múltiplos casos e foque em teste de teorias também pode ser considerado um estudo de caso. Segundo o autor, esta possibilidade de categorização não tem o objetivo de dar nomes diferentes para cada um dos desenhos resultantes das combinações, mas de fornecer uma forma diferenciada de pensar alternativas de formato dos desenhos de estudo de caso. Isso porque a forma como cada um desses seis elementos é incorporada no desenho vai resultar em uma forma diferente de conduzir o estudo de caso e, consequentemente, de analisar os dados coletados (DE VAUS, 2001).

A partir desta reflexão inicial sobre a questão da teoria, é muito importante destacarmos que há formas diferentes de tratá-la: seja dedutivamente ou indutivamente. Quando se tem um estudo de caso em que se pretende testar uma teoria, esta vai guiar quais observações podem ser consideradas evidências e como elas devem ser analisadas dentro do caso a fim de testá-la. Assim, a partir de uma teoria (uma proposição explicativa do fenômeno em questão), o estudo de caso testa sua capacidade de explicação dedutivamente, a partir das implicações observáveis coletadas no mundo real. Nesse sentido, o estudo de caso permite que, através de um maior número de observações dentro do caso escolhido, seja possível testar uma teoria em determinadas condições.

Ao contrário, quando se tem interesse em gerar uma teoria a partir da análise de um fenômeno, como o que está em questão, tem-se um processo indutivo, no qual a construção da proposição explicativa deve focar em compreender os mecanismos internos de funcionamento. Nessa perspectiva, para Creswell (2007), estu- 
dos de caso se enquadram numa forma de desenho de pesquisa qualitativa (do que nos diferenciamos na medida em que defendemos que é predominantemente, mas não exclusivamente, afinal é a densidade não o tipo de dado que caracteriza o estudo de caso). A pesquisadora explora um ou alguns sistemas delimitados dentro de um caso durante um período de tempo, de forma detalhada, com coleta de dados em profundidade e envolvendo múltiplas fontes, como entrevistas, observações, documentos. Logo, o foco está no desenvolvimento de uma descrição profunda e detalhada de um caso e suas dimensões (ou de suas subpartes, de seus processos internos, de suas conjunções causais, de sua variação intracaso) ou de alguns casos.

Além disso, esse tipo de desenho proporciona a utilização de métodos relativamente intensivos, de modo que um único caso pode questionar a validade de uma relação de causa e efeito (teoria) que foi estabelecida com base em muitas observações. Assim, os métodos orientados por casos fazem com que os pesquisadores considerem os casos como "entidades completas" e não como coleções de variáveis. Isto é: as diferentes partes e condições que formam um caso são entendidas relacionalmente, enquanto a pesquisadora é estimulada a dialogar ideias e evidências de modo que seja possível proporcionar o exame das diferentes formas de combinação das condições, em diferentes contextos e produzindo diferentes resultados (RAGIN, 1987)³.

Com tudo o que foi exposto, fica clara a visão mais ampla do que seja um estudo de caso, da qual compartilhamos. Ele deverá ser sempre constituíd o a partir de um conjunto de observações que possam ser utilizadas como evidências e que poderá ter diferentes unidades de análise, mas que tem a capacidade de nos ajudar a explicar a sua classe de eventos, como diz Gerring. Poderíamos, por um momento, apelidá-lo de "estudo de fenômeno", pois pode

\footnotetext{
3 De forma semelhante a esta perspectiva, Collier, Brady e Seawright (2004) atribuem distinções entre estudos observacionais que lidam com análises de bancos de dados daqueles que lidam com observações de processos causais, o que influencia na dimensão da força da inferência causal produzida pelo estudo. Afirma-se que, quando focados em observação de processos causais, estudos são mais propensos a se configurar com small- $n$, com níveis de mensuração nominais (mais baixos e com menos suposições sobre relações lógicas), analisando evidências relevantes sem recorrer frequentemente a testes estatísticos e com análises densas e detalhamento dos poucos casos. Este seria um modelo compatível com a definição de estudo de caso trazida por Gerring (2007), uma vez que a dimensão de observação de processos causais tem por objetivo explicitar a origem desse efeito na inferência causal, o que está ligada à longa tradição de análise within-case em pesquisa qualitativa.
} 
se restringir a um caso ou não, pode ser descritivo ou explicativo, pode partir de uma teoria (dedutivo) ou pode construir uma (indutivo), pode ser feito por uma abordagem qualitativa ou quantitativa, apesar de sempre visar a uma análise densa e intensiva de um fenômeno.

\section{Estudos de caso: possibilidades e desafios}

Diante da definição e das características apresentadas, seguimos para o tratamento dos desafios metodológicos, desenvolvendo as possibilidades referentes ao desenho de estudo de caso. Aqui trataremos especificamente da questão da multicausalidade dos fenômenos sociais, da seleção e número de casos para testar hipóteses e das implicações de validade inerentes aos desenhos de estudo de caso, entendidos em sua multiplicidade de significados e formatos, como já explicitado.

\section{Multicausalidade e complexidades dos fenômenos sociais}

A perspectiva da multicausalidade dos fenômenos sociais leva a uma série de questionamentos sobre os estudos de caso. A mais importante é como inferir resultados a partir da análise de um caso, dado que os fenômenos têm múltiplas causas, ou seja: como construir inferências válidas quando se tem mais variáveis do que casos para observar? Isso é importante porque um caso, representante do fenômeno a ser estudado, a princípio pode não garantir a variação necessária para a construção dessas inferências.

Para King, Keohane e Verba (1994), os estudos de caso de observação única não atendem aos propósitos de inferência causal porque a causalidade múltipla dos fenômenos sociais exige mais de uma observação para avaliar o impacto de variáveis explicativas. Além disso, a realidade social não é derivada de processos 
determinísticos, assim, o erro aleatório presente no mundo aparece mesmo se a mensuração for perfeita, o que raramente será no cenário de observação única. A partir disso, os autores se empenham em sugerir possibilidades de aumentar o $n$ (entendido como número de observações), pois quando ele é igual a "um" não é possível cumprir o critério da variação fundamental das variáveis dependente e independente, não sendo possível inferir causalidade. Não demorou muito para que diversos autores trouxessem críticas a essa visão, sustentando que existem diversas formas de conceituar estudos de caso (não apenas como observação única) e diferentes lógicas de causação, nas quais é possível sim, inferir causalidade através de estudos de caso (COLLIER, BRADY e SEAWRIGHT, 2004; BEACH e PEDERSEN, 2013) ${ }^{4}$.

Em nossa opinião, os autores têm razão e também não têm razão. Vejamos: eles têm razão quando afirmam que um só caso vai limitar a variação de sua dependente e esse pode ser um erro fatal. Quando isso está em jogo, é melhor seguir outra estratégia, pois as inferências a partir de um estudo de caso, de fato, serão muito limitadas. No entanto, na colocação dos autores há um estreitamento do que pode ser entendido como o fenômeno de interesse, pois desconsidera que há variações internas a um fenômeno que multiplicam "a" observação em muitas observações, seja no tempo ou no espaço, seja entre as dimensões que formam o fenômeno, seja nos casos que conformam o fenômeno central. Ou seja, há uma confusão entre os termos observação, caso e unidade de análise. Portanto, em sua obra seminal, os autores (KKV) trabalham com uma visão muito estreita do que pode ser e do que pode permitir um estudo de caso, mas não restam dúvidas do quanto eles obrigaram os "qualitativistas" a fazerem o dever de casa depois disso (mérito deles).

À multicausalidade acrescenta-se a ideia da complexidade de nossos fenômenos. Na perspectiva de Ragin (1987), sempre que se examina uma mudança de larga escala, é possível encontrar

4 Essa discussão começa no seminário realizado um ano depois da publicação de Designing Social Inquiry: Scientific Inference in Qualitative Research (1994), especificamente com o fim de discutir o livro, e se desenvolve até a publicação de Rethinking Social Inquiry: Diverse Tools, Shared Standards em 2004. 
combinações de condições que a produzem. Isso não é o mesmo que dizer que uma mudança resulta de muitas variáveis ${ }^{5}$. $\mathrm{O}$ argumento causal da combinação das condições diz respeito às suas intersecções em tempo e espaço, o que serve de base para o que Mill (1963) chama de "causação química": um fenômeno (ou uma mudança) ocorre apenas quando há uma intersecção de pré-condições apropriadas. Essa natureza conjuntural e de combinação é a principal característica da complexidade causal. Dessa forma, entende-se a complexidade dos fenômenos sociais não pela quantidade de variáveis explicativas, mas pela existência de condições causalmente relevantes que podem se combinar de formas diversas para produzir um determinado resultado.

Nesse sentido, a escolha dos casos é o primeiro desafio com o qual a pesquisadora tem que lidar ao optar por esse tipo de desenho. Creswell (2007) destaca a importância da escolha e delimitação do caso dentro do universo de possibilidades com que a pesquisadora se depara, de forma a determinar um objeto que seja digno de tal análise. A seleção do(s) caso(s) envolve o estabelecimento de um comportamento racional por parte da pesquisadora, para que possa agir de forma estratégica tanto na escolha quanto na coleta de informações sobre o(s) caso(s).

A quantidade e qualidade de informações disponíveis influenciam o valor que o estudo tem, pois afetam diretamente a profundidade da análise a ser realizada. Além disso, decidir os limites do caso em termos de tempo, eventos e processos é fundamental para determinar os limites da análise de forma adequada. Por fim, destaca-se o trade-off entre número de casos e profundidade da análise: quanto mais casos são incluídos, menos profunda será a análise; aqui Sartori nos alerta sobre qual nível de abstração é desejado em nossa análise, pois é isso que determinará essa escolha. Isso também implica em maior ganho em termos de comparação, sendo recomendado não ultrapassar quatro ou cinco casos para manter a característica fundamental de densidade e profundidade desse tipo de desenho (CRESSWELL, 2007).

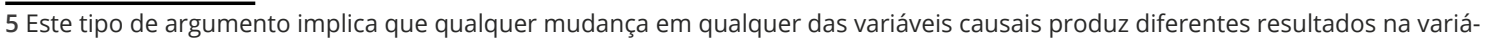
vel dependente (RAGIN, 1987). 
Ainda a respeito da seleção dos casos, Geddes (2006) trata de um dos principais desafios em estudos de casos específicos: o viés de seleção. A autora evidencia o problema da seleção com base na variável dependente e suas implicações na lógica explicativa, sendo possível identificar apenas diferenças entre os casos, sem concluir que qualquer fator comum aos casos revela uma causa, ou entender as relações entre os casos como universais. Para que haja contribuição no acúmulo de conhecimento teórico, portanto, deve-se selecionar os casos de modo a não diminuir ou desvalorizar a lógica de explicação. Geddes conclui mostrando que a escoIha do(s) caso(s) determina os resultados encontrados, logo não é possível justificar o caso apenas pelo interesse pessoal, proximidade ou algum tipo de facilidade, que são reais. É mandatório que o caso realmente lhe permita responder à questão proposta. Muitas vezes isso não acontece e o caso escolhido não se encaixa com a questão a ser respondida. Dessa forma, há que se ajustar: buscar um caso que responda à questão pretendida, se maior apego for à questão, ou buscar uma questão que possa ser respondida pelo caso que você quer estudar, se o apego for maior pelo caso.

Nesse sentido, cabe ainda destacar que os critérios para seleção de casos a serem estudados depende da forma que será realizada a análise empírica. Quando estamos falando em estudos quantitativos, a escolha de casos pela via da aleatoriedade é considerada como a melhor estratégia, a fim de garantir que não haverá vieses que irão comprometer a validade das inferências, que vão no sentido de evidenciar grandes tendências. Já quando estamos tratando de estudos com enfoque qualitativo, a escolha dos casos deve ser feita pela via da intencionalidade, pois não estamos interessados em evidenciar grandes tendências ou fazer inferências frequencistas (em termos estatísticos) para a população e para os casos, mas olhar, dentro de casos específicos, os mecanismos que se estruturam e levam à ocorrência do fenômeno de interesse.

Após a seleção dos casos, outra questão relacionada à multicausalidade dos fenômenos sociais é a dita impossibilidade de testar hipóteses a partir da utilização de apenas um caso, uma vez 
que, mesmo os estudos de caso com $n$ maior que um, o número de potenciais fatores causais é maior do que o número de casos da análise, então as evidências não podem corroborar ou negar uma hipótese. Dessa forma, Geddes (2006) destaca que, se apenas uma hipótese causal é considerada na análise, com apenas dois casos seria possível estimar as relações causais sugeridas por uma teoria. Entretanto, excluir causas possíveis que afetam o resultado e que estão correlacionadas não é a solução. Logo, a única solução real para este problema específico é aumentar o número de casos, como proposto por KKV (1994).

Desse modo, mais uma vez aparece o trade-off entre número de casos e profundidade da análise trazendo o questionamento: como aumentar o $n$ para testar relações causais sem perder as vantagens da densidade? A resposta está no aumento do número de observações dentro de um pequeno número de casos, sincronicamente (no espaço), olhando para regiões ou estados dentro do país, ou diacronicamente, olhando para períodos de tempo, por exemplo. Neste ponto, faz-se necessário destacar a importância de utilizar as mesmas categorias para atribuir valores às variáveis em todas as observações através de medidas que reflitam os mesmos efeitos causais potenciais da mesma forma para cada observação6 ${ }^{6}$ pois só assim é possível tirar conclusões válidas do estudo (GEDDES, 2006). A intenção é criar a variação interna em substituição à variação cross-case sobre a qual os autores falam. Portanto, é uma falácia a afirmação de que não é possível testar hipóteses a partir de um caso só, desde que se tenha um desenho que garanta a variação interna. Variação que é mais do que esse vocábulo indica, mas que se refere à complexidade do fenômeno a ser estudado.

Assim como nós, autores como Reuschemeyer (2003) defendem que estudos de caso históricos são capazes de muito mais do que simplesmente gerar hipóteses que serão testadas por estudos

\footnotetext{
6 Através da sistematização dos conceitos e operacionalização em indicadores que reflitam tais conceitos, é possível alcançar a validade de mensuração. Entretanto, para que estas medidas sejam confiáveis, faz-se necessário haver maior cuidado na coleta e formulação dos indicadores, de modo que estes sempre exibirão o mesmo score se a dimensão da realidade empírica a que se refere permanecer estática. Assim, o cuidado com a qualidade da informação, em todos os estágios pelos quais ela passa, é que pode garantir que mudanças observadas no indicador ao longo do tempo possam ser analisadas de forma consistente e confiável (JANNUZZI, 2003).
} 
large-n. Ele afirma que, além de desenvolver novas proposições explicativas, estes são também capazes de testá-las e até oferecer explicações causais persuasivas. O processo de teste de hipóteses por meio de poucos casos pode ocorrer por meio da falsificação de proposições teóricas não probabilísticas. Isto é, dada uma teoria determinística, que afirma "quando $X$, necessariamente, $Y$ ", e não havendo hipóteses rivais, confrontada com um único caso em que isso não se confirma, este único caso é capaz de falsear a teoria. Assim, visão cética sobre a afirmação de que um único caso pode testar uma teoria está baseada no erro de pensar em "caso único como observação única", de modo que

\begin{abstract}
uma boa análise histórica (com um considerável número de observações), analiticamente orientada, passa por frequentes iterações de proposições explicativas conflitantes com diferentes unidades de dados. Se esse confronto não acontece com o uso quantitativo de itens padronizados, mas tipicamente funciona no modo qualitativo, examinar diversas implicações da proposição explicativa interativamente envolve muitas verificações empíricas. E o ganho de credibilidade se dá exatamente pelo ajuste entre teorias e suas implicações complexas de um lado e melhores evidências empíricas do outro (REUSCHEMEYER, 2003, p. 318. Tradução livre'7).
\end{abstract}

\title{
A validade
}

A questão da validade é considerada central nas discussões acerca da importância dos desenhos de pesquisa na produção do conhecimento. Acompanhada de diversos adjetivos que determinam diferentes nuances do termo, a validade pode ser entendida, de modo geral, em três dimensões: validade interna, externa e de mensuração (SÁTYRO e REIS, 2014). Esta última está direta-

\footnotetext{
7 Original: "Good historical analysis that is analytically oriented goes through frequent iterations of confronting explanatory propositions with many data points. If this confrontation does not proceed with the quantitative use of standardized items but typically works in a qualitative way, examining many different implications of the explanatory propositions entertained, it nevertheless involves many such empirical checks. And it gains its credibility precisely from the fit between theoretical ideas and their complex implications, on the one hand, and the best empirical evidence, on the other" (REUSCHEMEYER, 2003, p. 318).
} 
mente relacionada à formulação sistematizada de conceitos, para que sejam operacionalizados de modo a desenvolver indicadores (quantitativos ou qualitativos) para mensurar ou classificar casos e estruturar a análise em qualquer desenho de pesquisa (ADCOCK e COLLIER, 2001). Entretanto, aqui iremos focar nos desafios de estabelecer validade interna e externa através do estudo de caso. Isso porque, para alcançar a objetividade na produção de conhecimento, é necessário agregar, em maior nível possível, a validade e confiabilidade da pesquisa.

De acordo com Gerring (2007), os estudos de caso tendem a priorizar a validade interna ${ }^{8}$ em detrimento da externa, uma vez que estudos cross-case são sempre mais representativos da população do que os estudos de caso, desde que se siga um procedimento de amostragem adequado. O problema da representatividade da amostra do estudo de caso ocorre porque há apenas um pequeno número de casos de um fenômeno mais geral, sendo, assim, mais fraco em validade externa. Inversamente, sua força da validade interna se dá pela possibilidade de identificar a relação causal no(s) caso(s) em análise por meio do estabelecimento dos mecanismos causais em sua complexidade e em sua multidimensionalidade.

Nesse sentido, estudos de caso podem adotar uma abordagem idiográfica ou nomotética ${ }^{9}$ para proporcionar explicações, apesar de, em geral, estarem relacionados à nomotética. Entretanto, com a primeira abordagem é possível alcançar uma compreensão contextualizada, a partir do uso de casos particulares para alcançar compreensões mais generalizáveis de uma proposição teórica mais ampla. Logo, ao desenvolver um entendimento causal a partir de uma perspectiva mais ampla, o estudo de caso pode atingir um alto nível de validade interna. Além de analisar a combinação de fatores causais particulares entre si, pode-se acessar a importância relativa dos casos particulares, mas também a forma como

$\overline{8 \text { A validade interna }}$ de um estudo vai depender da estrutura do desenho de pesquisa, possibilitando eliminar explicações alternativas e tirar conclusões que não são ambíguas a partir dos resultados encontrados, que respondem às questões de pesquisa (DE VAUS, 2001).

9 A abordagem de explicação nomotética permite focar em uma quantidade restrita de variáveis para oferecer explicações parciais para uma classe de casos, enquanto a abordagem idiográfica leva em conta um grande número de variáveis para oferecer uma explicação completa de um caso particular (DE VAUS, 2001). 
os vários casos se inter-relacionam, sem tirar as variáveis contextuais da análise. Dessa forma, os estudos de caso podem alcançar um entendimento sofisticado e equilibrado dos processos causais e, assim, evitam o erro de confundir correlação com causação (DE VAUS, 2001).

No que diz respeito à validade externa, reafirma-se o argumento de Yin (2001), ao diferenciarmos generalizações estatísticas de generalizações teóricas. É possível fazer uma generalização estatística quando se tem uma amostra aleatória representativa da população dos casos, generalizando com base na probabilidade estatística. Nessa perspectiva, não é razoável afirmar que estudos de caso oferecem a base para este tipo de generalização, uma vez que temos em mãos um ou poucos casos, escolhidos intencionalmente e estrategicamente para a análise, que não são necessariamente representativos da população. A generalização teórica, por sua vez, envolve o questionamento sobre o que o caso em análise nos diz sobre uma teoria específica, ou uma proposição teórica, com base na lógica de replicação, não de amostragem ${ }^{10}$. Um bom exemplo para isso continua sendo a forma como Robert Dahl desenvolveu as bases para "Polyarchy" (1971), a partir do estudo de um só município.

Os argumentos que destacam as dificuldades dos estudos de caso em produzir generalizações geralmente se apoiam na ideia de representatividade da amostra de small-n, como anteriormente discutido. Entretanto, para melhor elucidar essa questão cabe aqui também destacar as diferentes estratégias de análise causal e sua influência na possibilidade de generalização e da validade externa dos estudos de caso.

Basicamente, o que diferencia as estratégias de causação adotadas pelos estudos de caso e estudos cross-case é o nível de agregação. Ou seja, with-in case analysis implica comparar processos internamente a um caso específico, de modo a examinar múltiplas

\footnotetext{
10 Além disso, Kirk e Miller (1989) reforçam a importância da validade na medida em que a colocam, juntamente à confiabilidade, como sendo parte constitutiva da objetividade em estudos qualitativos. A confiabilidade corresponde ao grau em que o resultado encontrado não deriva de circunstâncias acidentais da pesquisa, ao passo que a validade é o grau em que o resultado é interpretado da maneira correta. Entretanto, a relação entre validade e confiabilidade não é simétrica, podendo haver perfeita confiabilidade sem validade, mas não o contrário. Ou seja, é preciso também falar em objetividade.
} 
O que é um Estudo de Caso e quais as suas potencialidades?

Natália Guimarães Duarte Sátyro•Raquel Wanderley D'Albuquerque

características do que originalmente se considera apenas um único caso para acessar hipóteses desenvolvidas em análises cross-case. Entretanto, para analisar os casos internamente, aplicam-se combinações das estratégias de mensuração que são comumente utilizadas em estudos cross-case (ordinal e nominal), as quais envolvem explicações de natureza probabilística e determinística11 (MAHONEY, 2003).

São muitas as técnicas que podem ser utilizadas em um estudo de caso. Em geral, os textos básicos tratam mais das técnicas de coleta de dados, como análise documental, entrevistas em profundidade, entrevistas estruturadas e semiestruturadas, grupos focais, entre outras. No entanto, a ênfase nessas técnicas consideradas mais qualitativas é um equívoco na medida em que é possível o uso de técnicas quantitativas12. Há algumas técnicas que são mais sofisticadas, que já são consideradas quasi-métodos, tais como análise de conteúdo e análise de discurso, por exemplo. No entanto, existem métodos que permitem o uso mais adequado dos dados colhidos e que permitem inclusive estabelecer relações causais. Dentre as possibilidades de acessar causalidade em estudos de caso, apresentamos aqui três lógicas13: comparação, congruência e rastreamento, que são também conhecidas como estudos de caso comparativos, pattern matching e process-tracing.

Os estudos de caso comparativos podem partir da premissa probabilística ou determinística para buscar regularidades nas relações entre os fatores explicativos através de análise de condições necessárias, suficientes e efeitos causais. Utiliza-se de contrafactuais, da lógica da eliminação ou da frequência para estruturar

\footnotetext{
11 Estratégias nominais e ordinais são utilizadas por estudos cross-case para estabelecer comparações. A diferença fundamental entre as duas é a lógica da inferência: a primeira tem um entendimento não linear de causação, construído com base na ideia de condições necessárias e suficientes. Já a segunda é mais compatível com suposições lineares e correlacionais de causação, tipicamente utilizadas em estudos large-n. Por sua vez, o entendimento sobre explicações e causação pode partir da lógica determinística ou probabilística. A primeira assume a existência de causas que apresentam, em determinadas condições, relações invariáveis entre os fatores de interesse, de modo que uma variação em $X$ necessariamente implica uma variação em $Y$, sendo esta última a variável dependente. Já a visão probabilística trata variáveis explicativas (ou combinações entre estas variáveis) como quase sempre necessárias ou suficientes para o entendimento da variável dependente, assumindo a possibilidade de aleatoriedade ou erro de mensuração presente na explicação (MAHONEY, 2003).

12 Seria muito restritivo enumerar algumas técnicas quantitativas possíveis uma vez que deverá ser a pergunta da pesquisa que guiará para um tipo de técnica ou outra, estando, portanto, um rol muito extensor à disposição. Para um exemplo clássico, lembramos de que Barry Ames (2003) em "Os Entraves da Democracia no Brasil" apresenta seu trabalho como um estudo de caso quantitativo, uma vez que se baseia em um large-n no sentido de muitas observações.

13 Um quarto método, a narrativa causal, consiste na combinação entre análises cross-case e with-in case, comparando casos de acordo com sequências de processos e eventos altamente desagregados, que levam a resultados. Este é um método que ainda carece de maior formalização (MAHONEY, 2003).
} 
estudos de caso contrafactuais, comparativos clássicos ou comparativos quasi-experimentais, respectivamente (BEACH e PEDERSEN, 2013).

Já as análises que se baseiam em pattern matching partem da prerrogativa determinística das explicações, buscando identificar regularidades e efeitos causais entre os fatores de interesses na análise. Aqui se analisam os padrões causais derivados das comparações entre casos que sugerem hipóteses adicionais sobre aspectos de casos específicos. Esta é uma das ferramentas que permitem testes de falsificações de teorias em uma pesquisa de small-n, uma vez que as observações internas aos casos são repetidamente consistentes com os achados nas análises entre casos, dando suporte à validade dos achados dessas últimas. Isso porque, dentre outros fatores, pattern matching ajuda a diminuir a quantidade de explicações possíveis ao proporcionar diferentes formas de eliminar variáveis, restando explicações muito mais parcimoniosas (BEACH e PEDERSEN, 2013; MAHONEY, 2003).

Por fim, o método do rastreamento, o process-tracing, pode ser entendido a partir do entendimento determinístico da causação que será inferida a partir da observação dos mecanismos causais, ou seja, busca-se identificar os links entre a(s) variável(is) dependente(s) e fatores explicativos. Esses mecanismos causais são definidos como os processos e variáveis intervenientes, através dos quais um fator explicativo exerce o efeito causal sobre a variável dependente. A grande força desse método está na possibilidade de eliminar o problema das relações espúrias que podem aparecer em análise de small-n, através da identificação das conexões entre as variáveis. Entretanto, ele não elimina fatores causais, mas dá suporte às explicações próprias. Nesse sentido, esse método supre uma das críticas feitas aos estudos cross-case de que a correlação identificada entre os casos não é causal, pois os mecanismos de ligação entre a variável explicativa e variável dependente não poderiam ser identificados (BEACH e PEDERSEN, 2013; MAHONEY, 2003). 
A partir destes esclarecimentos é possível identificar as diferentes formas de atribuir validade aos estudos de caso, pois a partir do entendimento determinístico ou probabilístico das explicações oferecidas, podemos inferir causalidade de maneiras distintas. Isso evidencia a capacidade de gerar e testar hipóteses, além de oferecer explicações a fenômenos sociais validamente através desse tipo de desenho (REUSCHMEYER, 2003). Sendo assim, acredita-se haver razões suficientes para dar mais crédito às inferências produzidas por estudos de caso.

Complementarmente, identificam-se recentes desenvolvimentos que atribuem maior validade aos métodos de estudo de caso, tais como os modos mais sistemáticos de teorizar e criar tipologias, que têm proporcionado melhores técnicas para a seleção de casos com o propósito de construção de teoria. Além disso, a literatura recente tem crescentemente defendido que, em certa medida, estudos de caso podem proporcionar bases para generalizações que vão além dos casos estudados, através de inferências sobre mecanismos causais e o entendimento dos tipos de frequência do contexto no qual os mecanismos estão presentes (BENNETT e ELMAN, 2006).

Ledo engano daqueles que consideram que estudos de casos se restringem ao uso de técnicas qualitativas ou são mais simples do que as técnicas quantitativas. Este texto não pretende desenvolver nenhuma das técnicas ou métodos apresentados, apenas oferecer uma ideia do leque de possibilidades. O objetivo disso é expandir a visão estreita que usualmente um iniciante desavisado apresenta: de que estudo de caso ou pesquisa qualitativa não precisa de aprofundamento metodológico.

\section{Exemplos de Estudos de Caso na Ciência Política}

Nessa seção, temos a intenção de trazer alguns exemplos de estudos de caso com o objetivo de mostrar a sua forte presença 
na disciplina, a complexidade de alguns e, ainda, a diversidade do seu uso. Mas os objetivos principais são, de um lado, ressaltar o fato de que nunca se trata de uma única observação e, por outro lado, dar uma ideia da variedade de técnicas quantitativas e qualitativas que podem ser utilizadas Tem o objetivo também mostrar que esse desenho de pesquisa vai aos extremos: permite desde inferências generalizáveis, construção de teorias, até a exploração de um aspecto dentro de um espaço pouco explorado pela teoria dominante, ou de algum campo de estudo em que as teorias ainda estão em desenvolvimento.

Quando falamos em estudo de caso na Ciência Política temos bons exemplos. Impossível não começar por "Who governs?", de Robert Dahl (2005), em que, a partir de um estudo sistemático das relações entre os grupos de poder em uma cidade, New Haven (Connecticut, EUA), ele constrói as bases para "Polyarchy" (1971), hoje considerado por muitos um clássico da teoria política contemporânea. É com ferramentas intrinsecamente qualitativas que o autor mostra a passagem de uma sociedade dominada por uma oligarquia tradicional para uma sociedade urbana industrial, realizando fortes inferências do que isso representou politicamente. Assim, é um estudo de caso de abordagem indutiva que mostra a transformação de um regime oligárquico para um sistema democrático pluralista. Dahl realiza a sua análise a partir do mapeamento da distribuição de recursos e dos padrões de influência em áreas distintas. A obra trabalha com uma conjunção de fatores que determinam a reconfiguração da cena política. Ou seja, a riqueza de um trabalho dessa natureza é a capacidade de entender como se dão os mecanismos causais que serão analisados em grande escala nos estudos de natureza quantitativa.

Outro exemplo é o trabalho de Robert Putnam (2000), o clássico "Comunidade e Democracia - a experiência da Itália moderna". Putnam realiza uma análise da vida político-institucional na Itália, do início da década de 70 ao final da de 80, englobando todas as 20 regiões, seu processo de formação e sua caracterização política, econômica e sociocultural. A tese central de Putnam é que o 
nível da comunidade cívica e do capital social de uma determinada região define o nível de seu desempenho institucional. Nessa análise, ele destaca que as diferenças nos padrões de cultura cívica e capital social que caracterizam o norte e o sul italianos determinam a eficácia no primeiro caso e a ineficácia no segundo caso. Para explicar essas distinções culturais e de desempenho institucional, o autor usa uma série de diferentes técnicas para cada parte de sua pesquisa, dados temporais, surveys, entrevistas variadas, construção de indicadores, entre outras.

Poderíamos agregar a Dahl e Putnam o emblemático livro de Robert Michells (1962), o clássico "A Lei de Ferro das Oligarquias", ou o clássico de políticas sociais nos Estados Unidos, "Protecting mothers and soldiers" de autoria de Theda Skocpol (1995), como exemplos de trabalhos que se preocuparam tanto com a validade interna quanto com a externa a partir de desenhos de estudo de caso.

Mas não são somente os clássicos que podem ser considerados bons exemplos de estudo de caso. Escolhemos duas teses defendidas recentemente, nas quais foram utilizadas técnicas de process tracing e análise de rede para a construção de suas inferências. Uma leva o título "Redes de influência, burocracia, política e negócios na Agência Nacional de Saúde Suplementar (ANS)", de autoria de Marcello Baird (2017). O trabalho analisa as disputas de poder entre sanitaristas e liberais que são transpostas para o interior da Agência Nacional a partir das nomeações políticas para os cargos de direção e mapeia a forma como isso influenciou a regulação do setor na última década e meia.

O segundo trabalho, de autoria de Iris Gomes dos Santos (2015), intitulado "Agentes de Mudança, contextos políticos e dinâmica institucional: um estudo sobre o processo de mudança institucional gradual no setor da segurança pública no Brasil", mostra mudanças em uma área em que a maioria da literatura defende que há estabilidade. $O$ método de process tracing e análise de rede, assim como no trabalho de Baird, permitiu resultados sólidos que demonstram que houve uma trajetória de conversão gra- 
dual dentro das regras rígidas, mesmo na presença de muitos atores de veto. A criação de regras em nível intermediário preencheu lacunas constitucionais que permitem falar de mudanças graduais na política de segurança pública.

Por fim, é possível achar muitos exemplos de estudos de caso também em artigos, trabalhos sérios que superam um vício antigo da área de se restringir a ensaios. Nesse caso, devido ao espaço, as pretensões em geral são menores, mas nem por isso, menos importantes. Um exemplo é o estudo de caso denominado "Can the claim to foster broad participation hinder deliberation?", de Ricardo Mendonça e Eleonora Cunha (2014), que traz uma análise sobre o Legislativo do estado de Minas Gerais. Com o artigo aprende-se tanto sobre o assunto quanto sobre o uso de entrevistas, análise documental e grupo focal como ferramentas de levantamento de evidências que dão suporte à narrativa.

Outro tipo de estudo de caso é aquele que usa técnicas quantitativas, como é o caso do artigo "Análise espacial da burocracia da assistência social nos municípios brasileiros: notas para uma reflexão sobre a capacidade de implementação dessa política", de Natália Sátyro, Eleonora Cunha e Járvis Campos (2016). Os autores entendem que há padrões espaciais na variação da estrutura burocrática municipal que não estão devidamente explorados e isso justifica um estudo exploratório que ajude na elaboração de perguntas para o campo. O estudo parte de uma hipótese de que há uma espacialidade não randômica no fenômeno estudado. Assim, através do uso de análise espacial, os resultados mostram que, ao contrário das hipóteses clássicas, no nível municipal a capacidade de implementação e da gestão da política de assistência social independe do tipo de vínculo dos profissionais e da existência de uma burocracia estável e profissionalizada. Os autores reconhecem as restrições do trabalho, mas abrem uma agenda de pesquisa.

Um trabalho publicado no Caderno $\mathrm{CRH}$, intitulado "Comparando políticas sociais em governos de esquerda", de Sátyro et al (2019), apresenta um exemplo de desenho que integra uma análise cross-case com within-case. Os autores utilizam as duas estratégias de 
pesquisa para aumentar a variância de seu fenômeno. Comparando três políticas, tanto entre os três países analisados como para cada país, eles realizam estudos longitudinais que permitem a comparação com governos anteriores aos do foco do trabalho.

Esses exemplos têm o propósito de mostrar como um estudo de caso pode demandar uma variedade de técnicas para buscar evidências válidas para todos os mecanismos e processos analisados, mas que nenhum se restringe a uma observação ou uma evidência. Em um primeiro momento, traríamos apenas exemplos clássicos que permitem mostrar a força teórica dos estudos de casos. No entanto, a decisão final foi trazer exemplos mais simples, trabalhos inclusive passíveis de críticas e questionamentos, mas que também se aproximem mais do leitor mediano do que um estudo clássico. Os últimos exemplos permitem mostrar ao leitor tanto a existência de diferentes formas e estratégias de utilização deste desenho na Ciência Política, quanto a possibilidade de se executar estudos interessantes e exequíveis no período de um mestrado ou doutorado, a partir de um pensamento metodologicamente disciplinado.

\section{Considerações finais: em defesa dos Estudos de Caso}

Por fim, e diante do avanço da tendência de abordagens quantitativas, é importante realizar a defesa aberta do papel ocupado pelos estudos de caso. Primeiramente, é através de estudos de caso que podemos atingir níveis mais altos de validade conceitual ou identificar e estabelecer indicadores que melhor refletem os conceitos que pretendem ser medidos (validade de mensuração). É possível ainda haver maiores níveis de refinamento, uma vez que o pequeno número de casos corre menos riscos de estiramento conceitual (SARTORI, 1970).

Há uma vantagem comparativa nos estudos large-n na identificação de novas variáveis e hipóteses explicativas, através da 
análise de casos desviantes ou outliers, pela lógica de pensamento indutiva no tratamento do campo de implicações observáveis da teoria. Nesse sentido, também se destaca a possibilidade de examinar detalhadamente a operação de mecanismos causais em casos individuais, de modo a observar um grande número de variáveis intervenientes e observar aspectos inesperados da operação de tais mecanismos, além de identificar as condições presentes que os ativam. Isso tudo associado à habilidade de acomodar relações causais complexas na análise, tais como equifinalidade, efeitos de interação complexos e dependência de trajetória (path dependency). Isso leva a generalizações de menor alcance via teoria de médio porte, menos vagas e mais contextuais (GEORGE e BENNETT, 2004).

Finalmente, comungamos a ideia de autores que acreditam haver razões para defender os estudos de caso na Ciência Política. Para Geddes (2006), os estudos de caso têm seu valor e seu lugar na Ciência Política comparada, uma vez que através deles é possível fazer estudos detalhados de fenômenos, identificar plausíveis variáveis causais e trazer para o campo de estudos as exceções que não são englobadas pelas teorias explicativas, apesar de não poder testá-las. Já de acordo com Rezende (2011), os estudos de caso, que eram desenhos considerados problemáticos e altamente criticados no que se refere à inferência causal, assumem um papel importante com possibilidades metodológicas frutíferas para compreender processos causais interligados aos fenômenos dentro da realidade estudada. Isso é devido à sofisticação e ao esclarecimento metodológico dos pontos já discutidos sobre a validade, seleção de casos, problemas de generalização e diferentes estratégias de acessar e inferir causalidade. Estes desenhos se afastam da realidade de limitações metodológicas, produzindo generalizações e conhecimento.

É possível apreender, portanto, que a discussão acerca dos desenhos de pesquisa é essencial para que deixemos de lado visões estreitas e imprecisas acerca de suas capacidades de produção de conhecimento científico. Nesse sentido, defendemos que os es- 
tudos de caso correspondem a uma estratégia de pesquisa cujo valor e complexidade ainda precisam ser evidenciados e reconhecidos no âmbito da Ciência Política brasileira. Aqui, como dito na introdução, não houve intenção de trazer uma inovação ao campo, mas de apresentação de breve estado da arte para iniciantes e curiosos. Com a revisão conceitual, abarcando possibilidades, desafios e exemplos de seu uso, fica claro que grandes contribuições teóricas e empíricas do nosso campo de estudo derivam desta estratégia de pesquisa e, portanto, não podemos relegar a segundo plano o seu rigor metodológico.

\section{Referências}

ADCOCK, Robert; COLLIER, David. Measurement validity: a shared standard for qualitative and quantitative research. American Political Science Review, v. 95, N. 3, P. 529-546, 2001.

AMES, Barry. Os entraves da democracia no Brasil. Rio de Janeiro: Ed. FGV, 2003.

BAiRd, Marcelo. Redes de influência, burocracia, política e negócios na Agência Nacional de Saúde Suplementar (ANS). Tese defendida no Programa de Pós-Graduação em Ciência Política da Universidade de São PAULo, 2017.

BeaCh, Derek; Pedersen, Rasmus. Process-tracing methods: foundations and guidelines. Ann Arbor (MI): University of Michigan Press, 2013.

BenNetT, Andrew; ElMAN, Colin. Qualtative research: recent developments in case study methods. Annual Review of Political Science, v. 9, p. 455-476, 2006.

COllier, David; Brady, Henry; SeAWRight, Jason. Sources of leVerage IN CAUSAl INFERENCE: TOWARD AN ALteRnAtive VIEW OF METHodology. IN: 
Brady, Henry e Collier, David. Rethinking social inquiry: diverse tools, SHARED STANDARDS. LANHAM (MD): RowMAN AND LITTLEFIELD, 2004.

CRESWELL, John. Qualitative InQUiRY AND RESEARCh DESIGN: CHOOSING among five traditions. Thousand Oaks (CA): Sage Publications, 2007.

DaHL, Robert. Polyarchy: participation and opposition. New Haven (CO): YALE UniVERSITY PRESS, 1971.

DAHL, Robert. Who governs? Democracy and power in an American city. New Haven (CO): Yale University Press, 2005.

DE VAUS, David (Ed.). Research design in social research. Thousand Oaks (CA): Sage Publications, 2001.

GeDDes, Barbara. Paradigms and sand castles: theory building and RESEARCH DESIGN IN COMPARATIVE POLITICS. AnN ARbor (MI): University of Michigan Press, 2006.

GeOrge, Alexander; BenNetT, Andrew. Case studies and theory development in the Social Sciences. Cambridge (MA): MiT Press, 2004.

GERRING, John. The CASE Study: What it IS AND What It does. In: BoIX, Carles e Stokes, Suzan C. The Oxford Handbook of Comparative Politics. Oxford e Nova York: Oxford University Press, 2007.

JaNNUZZI, Paulo. Indicadores sociais no Brasil. Conceitos, fontes de DADOS E APLICAÇÕES PARA FORMULAÇÃO E AVALIAÇÃO DE POLÍTICAS PÚBLICAS E elaboração de estudos socioeconômicos. Campinas: Editora Átomo e Alínea, 2003.

KING, Gary; KEOHANE, Robert; VERBA, Sidney. Designing social inquiRy: scientific inference in qualitative Research. Princeton (NJ): Princeton UNIVERSITY PRESS, 1994.

KIRK, JeRome; MILLER, Marc. Reliability and validity in Qualitative Research. Thousand Oaks (CA): Sage Publications, 1989. 
MAHONEY, James. StRATEgIES Of CAUSAL ASSESSMENT IN COMPARATIVE HISTORICal analysis. In: Mahoney, James e Rueschemeyer, Dietrich. Comparative historical analysis in the social sciences. Nova York e Cambridge: Cambridge UNIVERSITY PRESS, 2003.

MENDONÇA, Ricardo; CUnHA, Eleonora. Can the claim to foster broad participation hinder deliberation? Critical Policy Studies, v. 8, N. 1, P. 78$100,2014$.

MiChels, Robert. Political parties: a sociological study of the oligarCHICAL TENDENCIES OF MODERN DEMOCRACY. NOVA YoRK: DoVER, 1962.

MiLL, John Stuart. A system of logic. In: ROBSON, J. M. (Ed.). The collected works of John Stuart Mill. Toronto: University of Toronto Press; London: Routledge and Kegan Paul, 1963-1991. 33 vols. Vol. VII.

PUTNAM, Robert. Comunidade E democracia: A EXPERIÊNCIA DA ITÁLIA MODerna. Rio de Janeiro: Ed. FGV, 2000.

RAGiN, Charles. The comparative method: moving beyond qualitative and quantitative strategies. Berkeley (CA): University of California Press, 1987.

REZENDE, Flávio. Razões Emergentes para a validade dos estudos de CASO na Ciência Política comparada. Revista Brasileira de Ciência Política, n. 6 , P. 297-337, 2011.

ReZende, Flávio. O pluralismo inferencial na Ciência Política pós-KKV (2005- 2015): argumento e evidências. Revista Política Hoje, v. 26, N. 1, P. 241-277, 2017.

RUESCHEMEYER, Dietrich. CAN ONE OR A FEW CASES YIELD THEORETICAL gains?. In: Mahoney, James e Rueschemeyer, Dietrich. Comparative historical analysis in the social sciences. Nova York e Cambridge: Cambridge UNIVERSITY PRESS, 2003.

SANTOS, Íris. Agentes de MUdANÇA, contextos polítıcos E diNÂMICA INSTITUCIONAL: UM ESTUDO SOBRE O PROCESSO DE MUDANÇA INSTITUCIONAL GRADUAL NO 
O que é um Estudo de Caso e quais as suas potencialidades?

Natália Guimarães Duarte Sátyro • Raquel Wanderley D’Albuquerque

Setor da Segurança pública no Brasil. Tese (Programa de Pós-Graduação em Ciência Política da Universidade Federal de Minas Gerais), 2015.

SARTORI, Giovanni. Concept misinformation in comparative politics.

American Political Science Review. v 64, N. 4, P. 1033-10553, 1970.

SÁtYro, NatÁlia; REIS, BRUNO. REFLEXões SOBRE A PRODUÇão dE INFERÊNCIAS INDUTIVAS VÁlIdAS EM CIÊNCIAS SOCIAIS. TeORIA \& SocIedade, v. 22, N. 2, P. 1339, 2014.

SÁtYro, Natália; CUNHA, Eleonora; CAMPOS, JárVIs. Spatial analysis of SOCIAL ASSISTANCE BUREAUCRACY IN BRAZILIAN MUNICIPALITIES: NOTES FOR REFLECtion on the IMPlementation Capacity of SOCIAL-Assistance Policy. Opinião Pública, v. 22, N. 2, P. 286-317, 2016.

SÁTYRo, Natália; FREITAS, Rosiene; ZANETTI, DanúBia; BARBOSA, Pedro. Comparando políticas socials em governos de esquerda. Caderno CRH, SalvadoR, v. 32, N. 85, P. 125-148, 2019.

SKOCPOL, Theda. Protecting soldiers and mothers. Cambridge (MA): Harvard University PRESS, 1995.

Yin, Robert. K. Estudo de caso. Planejamento e métodos. Porto Alegre: BOOKMAN, 2001. 